\title{
Schwerpunkt: Schelling zwischen Metaphysik und Erfahrung der Freiheit
}

DOI 10.1515/dzph-2017-0014

In den letzten zwei oder drei Jahrzehnten haben sich das Ansehen und der Ruf nachkantischer deutscher Philosophie in der englischsprachigen Welt dramatisch verändert. Nachdem der Deutsche Idealismus für den größten Teil des 20. Jahrhunderts, im Anschluss an Russells und Moores Angriff auf den viktorianischen Hegelianismus, als Musterbeispiel für altmodische und verrufene Spekulation galt, sind seine Themen und Erkenntnisse inzwischen als bedeutende Momente in die gegenwärtige philosophische Diskussion zurückgekehrt. Während manche bekannte Philosophen wie Robert Brandom und John McDowell sich in ihrem eigenen Werk auf die Innovationen der Deutschen Idealisten - besonders Hegels - bezogen haben, haben andere wie Robert Pippin und Terry Pinkard neue Interpretationen der Idealisten selbst entwickelt, die die Einschlägigkeit ihrer Anliegen betonen und sie ins Gespräch mit dem Mainstream bringen sollen. Gleichzeitig hat es ein Aufkeimen eher historisch orientierter Arbeiten über die vielfältigen Entwicklungen in der deutschen Philosophie gegeben, die durch die Veröffentlichung von Kants drei Kritiken ausgelöst worden waren. Es ist zweifelhaft, ob es vor ein paar Jahrzehnten überhaupt viele englischsprachige Philosophen gegeben hat, die je vom Pantheismusstreit gehört hatten, geschweige denn wussten, worum es dabei ging. Heute gibt es eine Überfülle an Büchern und Artikeln, aus denen sie es lernen können, begonnen mit Frederick Beisers The Fate of Reason, das in diesem Jahr seinen 30 . Geburtstag feiert. ${ }^{1}$ Es fällt allerdings auf, dass bis heute das Denken Schellings - anders als jenes Hegels und Fichtes in dieser anglophonen Idealismusrenaissance fast keine Rolle gespielt hat. ${ }^{2}$ Bei näherem Nachdenken scheint diese Lücke jedoch nicht allzu schwer zu erklären.

Es gibt viele Schilderungen, die entworfen werden können, um die Rehabilitierung des Deutschen Idealismus in der englischsprechenden Welt zu erklären. Nach einer plausiblen Lesart war allerdings die Veröffentlichung von Henry Allisons Kant's Transcendental Idealism 1983 ein wichtiger Wendepunkt. Eine von Allisons Hauptleistungen war es nämlich, den transzendentalen Idealismus als eine ernsthaft $\mathrm{zu}$ diskutierende philosophische Position wiederzubeleben.

\section{Beiser (1987).}

2 Erst vor drei Jahren ist die erste Sammlung englischsprachiger Essays von führenden Experten für den Deutschen Idealismus, die sich ausschließlich mit Schelling beschäftigt, erschienen (Ostaric 2014). 
Dies war offensichtlich auch eine Vorbedingung für die Welle neuer historischer Studien im Bereich der nachkantischen Philosophie, denn wenn man den transzendentalen Idealismus nicht wenigstens als eine vertretbare Option ansieht, bleiben die Debatten und Theorieentwicklungen, die von der Veröffentlichung der Kritik der reinen Vernunft ausgelöst wurden, wenig interessant oder relevant. Gleichzeitig machte Allisons Interpretation von Kants Idealismus das Bild Kants als eines Denkers populär, der sich mit der Beziehung zwischen zwei Standpunkten (einem hypothetischen absoluten und dem des endlichen Erfahrungssubjekts) beschäftigte und eigentlich kein Metaphysiker war, der zwischen der Welt der Sinne und einer intelligiblen Welt hin- und herpendelte.

Mit diesem Kompass in der Hand konnte das Gelände des nachkantischen Denkens auf neue Weise erkundet werden. Fichte, zumindest der Fichte der 1790er, der hauptsächlich von Interesse war, kann ohne Mühe innerhalb des „Zwei-Standpunkte“-Auslegungsrahmens gelesen werden. Er lässt sich sogar als der erste Vertreter einer nachdrücklichen Version dieses Ansatzes ansehen. Denn Fichtes Werk der Jenaer Periode ließ den metaphysischen Rest des Dings an sich fallen und konzentrierte sich auf die Beziehung zwischen der Erfahrung des Subjekts von sich selbst als konfrontiert mit einer determinierten, objektiven Welt einerseits und als eine spontane, selbstbestimmte Tätigkeit andererseits. Wegen seiner Ablehnung unaufgelöster Dualismen mag Hegel als schwieriger mit dieser Perspektive in Einklang zu bringen erscheinen. Wenn man jedoch annimmt, dass sein Terminus „Geist“ sich im Kern auf die normative Struktur der sozialen Welt voneinander abhängiger menschlicher Akteure bezieht, dann lässt sich auch auf Hegel eine Version des Zwei-Standpunkte-Rahmens anwenden. Hierbei wird jedoch der absolute Standpunkt, wenn auch nur als bereitstehende Option, die Ontologie des wissenschaftlichen Naturalismus, der als eine gültige Darstellung der materiellen Realität anerkannt wird, aber dann als irrelevant beiseitegeschoben wird, zumindest, soweit es um die Erfahrung unserer selbst als praktische Akteure, die die menschliche Lebenswelt bewohnen, geht. Robert Pippin fasst diese Sicht auf Hegel, die er vertritt, zusammen, indem er dessen Beschreibungen des Geistes als progressiver Prozess der Ablösung von der Natur stark macht. ${ }^{3}$

3 Pippin zitiert oft Hegels Aussage aus seinen Vorlesungen zur Philosophie des subjektiven Geistes: „[D]ie Natur des Geistes ist diese absolute Lebendigkeit, dieser Prozess selbst zu sein, von der Natürlichkeit Unmittelbarkeit zugehen, seine Natürlichkeit aufzuheben zu verlaßen, und zu sich selbst zu kommen, und sich zu befreien“ (Hegel 1978, 6). Er liest dies so, dass ,at a certain level of complexity and organization, natural organisms come to be occupied with themselves and eventually to understand themselves in ways no longer appropriately characterized within the boundaries of ,nature““ (Pippin 1999, 201 u. 197). Pippin übersieht dabei, dass nach Hegel der Geist die eigene Natürlichkeit aufhebt und daher schon im Natürlichen am Werke war. 
Die philosophische Strategie hinter diesem Interpretationsansatz wird von Terry Pinkard mit bewundernswerter Klarheit im Fichte-Kapitel seiner Geschichte des Deutschen Idealismus und seiner Folgen erläutert:

From the physical standpoint, saying „A = A“ is just causing sound waves to be sent through the air; it is only from the normative standpoint that it can be taken to mean anything. (Signing a check, hitting a home run, making an assertion, shopping at a sale are all other examples of normative activities that cannot be captured in a purely physical or „naturalistic" description of them.) Such statuses must therefore be instituted and not, as it were, discovered in the world. As such they cannot be „facts“ in any ordinary sense. ${ }^{4}$

Das Problem hier ist, dass der normative Standpunkt einen ausschlaggebenden Unterschied dazwischen, wie ein Zustand sein sollte und wie er tatsächlich ist, benötigt (auch wenn diese Diskrepanz in keinem Einzelfall instanziiert zu werden braucht). Nun behauptet Pinkard nicht, dass die von ihm erwähnten normativen Tätigkeiten ontologisch mehr sind als die physischen Vorgänge wie er betont, gibt es keine zusätzlichen „Fakten“. ${ }^{5}$ Physische Vorgänge lassen jedoch keine Unterscheidung dazwischen, wie sie tatsächlich vor sich gehen und wie sie es sollten, zu; daher müsste der normative Standpunkt eine Illusion sein. Anders formuliert: Pinkard behauptet, dass normativer Status instituiert werden muss. Dies impliziert, dass eine Zeit vor der Institution war: eine Zeit, zu der es nur physische Vorgänge gab. Die Frage ist dann: Wie können physische Vorgänge mit ihren notwendigen kausalen Wechselwirkungen je eine Norm hervorbringen - ein regulatives Prinzip, an das man sich halten kann oder nicht? Dieses Ereignis in die Nebel der Vorgeschichte zurückzuverlegen, trägt nichts zur Lösung des philosophischen Problems bei.

Es ist hier ironisch zu bemerken, dass die Überzeugung, das Normative sei toto caelo vom Natürlichen getrennt, als eine der zentralen philosophischen Annahmen gelten kann, denen sich der Deutsche Idealismus entgegenstellte. Man könnte sogar so weit gehen zu behaupten, dass diese Gegnerschaft und die entsprechenden Versuche, Natur und Normativität systematisch zu integrieren, ohne das eine auf das andere zu reduzieren, eines der Erkennungsmerkmale des Deutschen Idealismus ist. Die Ironie wird dadurch verstärkt, dass anderswo in der gegenwärtigen anglophonen Szene Philosophen die Kraft von Argumenten wiederentdeckt haben, die die Trennung zwischen Natur und Norm, zwischen

4 Pinkard (2002), 113, Hervorh. im Orig.

5 Pinkards Ergänzung „in any ordinary sense“ scheint ambivalent. Es ist schwer abzustreiten, dass es normative Fakten gibt, aber dies offen zuzugeben stünde in Spannung mit Pinkards Versuch, einen ontologiefreien Bereich der Normativität zu etablieren. 
physisch Objektivem und Subjektivem, infrage stellen, aber dies ohne jede nennenswerte Bezugnahme zu den nachkantischen Präzedenzfällen. ${ }^{6}$

Angesichts der Eigenart dieser jüngsten Rezeptionsgeschichte sind wir nun imstande zu verstehen, warum es so lange gedauert hat, bevor Schelling begann, in den gegenwärtigen englischsprachigen Diskussionen einen angemessenen Platz einzunehmen. Fichtes Werk der 1790er bleibt nämlich offensichtlich dem kantischen Primat des Praktischen verpflichtet, während Hegels oberste Kategorie - „Geist“ - sich gleichfalls zu einer starken praktischen Interpretation eignet. Schelling hingegen widersetzte sich seit dem Beginn seiner philosophischen Karriere der Unterordnung des Ontologischen unter das Praktische oder Normative - wobei er natürlich das Gespür für die Notwendigkeit, beide letztendlich systematisch zu versöhnen, nie verlor. In einem seiner frühesten veröffentlichten Texte, Vom Ich als Prinzip der Philosophie, schreibt er beispielsweise „Gebt dem Menschen das Bewusstsein dessen, was er ist, er wird bald auch lernen, zu sein, was er soll: gebt ihm theoretische Achtung vor sich selbst, die praktische wird bald nachfolgen. “7 Es kann gut sein, dass aus Schelling entnommene Überlegungen sich als nützlich erweisen werden, um den derzeitigen Wildwuchs der puren Normativität zu bekämpfen, ohne in reduktionistische Formen des Naturalismus zurückzufallen.

Die vier in diesem Schwerpunkt veröffentlichten Artikel behandeln alle in verschiedener Weise das Verhältnis von Ontologie und Normativität bei Schelling. Sebastian Gardner schlägt eine Interpretation von Schellings berühmtestem Text, der Abhandlung Über das Wesen der menschlichen Freiheit von 1809, als eines komplexen, vielschichtigen Versuchs vor, von Kants Theorie menschlicher Freiheit hinterlassene Probleme zu lösen. Aus Gründen, die aus meinem oben vorgetragenen Argument hervorgegangen sein sollten, kritisiere ich Gardners Vorschlag, demzufolge Schelling die Ontologie der Freiheitsschrift ausschließlich vom Standpunkt des praktischen Bewusstseins her konstruiere, und gleichermaßen eine frühere Interpretation des Texts durch Markus Gabriel, der mir in den

6 Sacks (2000) entwickelt im Ausgang von Kant im Alleingang eine drastische Form des transzendentalen Idealismus, jedoch völlig ohne sich auf das fichtesche Beispiel zu beziehen. Nagel (2012) schlägt eine teleologische, wertegeleitete Konzeption der Natur vor; er beschreibt sich selbst als einen „objektiven Idealisten“, aber er lässt nur einmal Hegel und Schelling leise anklingen (ebd., 17). Strawson (2006) verteidigt, was er einen „equal-status fundamental-duality monism“ nennt (ebd. 241), mit üppigen Bezügen auf historische Vorgänger, aber ohne jedwede Nennung von Schelling. Demnach könnte seine Aussage, dass ,although there is no non-experiential being absolutely speaking, there is non-experiential being relatively or relationally speaking“ (ebd., 261), fast Wort für Wort aus Schelings Identitätsphilosophie stammen.

7 Schelling (1980), 77-78, Hervorh. im Orig. 
entgegengesetzten Irrtum zu verfallen scheint. Gardner hat eine erhellende Replik auf meine Kritik geschrieben, die ebenfalls hier erscheint. Abschließend betrachtet Teresa Pedro die Verbindungen zwischen Schellings Philosophischen Briefen über Dogmatismus und Kritizismus und dem Pragmatismus von William James. Pedros Aufsatz mag die Position zu vertreten scheinen, dass Schelling - zumindest zu bestimmten Zeitpunkten seiner Entwicklung - willens war zu verneinen, dass die Wahrheit der Ontologie sich von unserer praktischen Gerichtetheit auf die Welt ablösen lasse. Wenn man jedoch davon ausgeht, dass Schellings grundlegende Behauptung in den Briefen besagt, dass die absolute Realität dadurch charakterisiert ist, dass man sich ihr stets nur aus der einen oder anderen praktischen Perspektive nähern kann, scheint diese Schlussfolgerung jedoch nicht mehr so zwingend.

Aus dem Englischen von Matthias Warkus

Peter Dews, peted@essex.ac.uk

\section{Literatur}

Beiser, F. (1987), The Fate of Reason: German Philosophy from Kant to Fichte, Cambridge, Mass. Hegel, G. W. F. (1978), Hegels Philosophie des subjektiven Geistes, hg. v. Petry, M. J., Dordrecht u. Boston.

Nagel, T. (2012), Mind and Cosmos, Oxford.

Ostaric, L. (Hg.) (2014), Interpreting Schelling: Critical Essays, Cambridge.

Pinkard, T. (2002), German Philosophy 1760-1860, Cambridge.

Pippin, R. (1999), Naturalness and Mindedness: Hegel's Compatibilism, in: European Journal of Philosophy 7.2, 194-212.

Sacks, M. (2000), Objectivity and Insight, Oxford.

Schelling, F. W. J., (1980), Vom Ich als Prinzip der Philosophie oder über das Unbedingte im menschlichen Wissen, in: Historisch-kritische Ausgabe I,2, Stuttgart, 67-176.

Strawson, G., et al. (2006), Consciousness and its Place in Nature, hg. v. Freeman, A., Charlottesville, Va., u. Exeter. 